\title{
Sustained vigilance and animal performance
}

\author{
REUVEN DUKAS \& COLIN W. CLARK \\ Institute of Applied $M$ athematics, U niversity of B ritish Columbia, Vancouver, B ritish Columbia \\ V6T 1Z2, Canada \\ ( Received 14 J anuary 1994; initial acceptance $22 \mathrm{M}$ arch 1994; \\ final acceptance 7 M ay 1994; M S. number: ${ }^{46900)}$
}

\begin{abstract}
Psychological studies have established that the central nervous system cannot sustain vigilance for an extended period of time. The consequent vigilance decrement implies a gradual reduction in the ability to process information effectively. This may result in a reduced ability to detect hidden predators, locate cryptic food items or make successful behavioural decisions. A model incorporating parameters of vigilance decrement during foraging and vigilance recovery during rest predicts first, the optimal allocation of time between foraging activity and rest, and second, the optimal length of each foraging episode. F or a given rate of vigilance recovery, the model predicts that both the optimal proportion of time devoted for foraging activity and the optimal length of a foraging bout should be decreasing functions of the rate of vigilance decrement. Because the rate of vigilance decrement is larger for more difficult tasks, both the total time spent foraging and the duration of each foraging episode should be smaller for more demanding foraging activities. It is suggested that vigilance decrement is a dominant factor determining temporal patterns of animal behaviour.
\end{abstract}

One of the fundamental limitations of the central nervous system is its inability to sustain a high quality of information processing for an extended period of time. Consequently, performance on tasks such as the detection of cryptic targets degrades over time; this gradual reduction in performance is more pronounced for more difficult tasks (N uechterlein et al. 1983; W arm 1984; Parasuraman \& M ouloua 1987). In psychological terms, subjects cannot sustain vigilance, or attention, while engaged in a difficult cognitive task. Here 'vigilance' is used in its broad sense to imply a state of alertness directed towards certain stimuli; a higher level of vigilance enables the brain to better process and respond to these stimuli (M ackworth 1969; M ackie 1977; Parasuraman 1984).

Theoretical, empirical and applied aspects of sustained vigilance have been widely studied for human performance (M ackworth 1948; Davis \& Parasuraman 1982; Wickens 1984). In contrast, studies of non-human subjects have been restricted to neurobiological research (F oote et al. 1980; A ston-J ones et al. 1984). N evertheless, the problem of sustained vigilance is highly relevant for the daily patterns and types of activity conducted by animals in their natural settings (D imond \& Lazarus 1974).

0003-3472/95/051259+09 \$08.00/0
To further evaluate the importance of sustained vigilance for animal performance, we present here a set of simple models. These models predict first, the optimal allocation of time between foraging activity and rest, and second, the optimal length of each foraging episode.

\section{MODELS AND PREDICTIONS}

Throughout this paper, we refer to 'vigilance' in its more general meaning usually used in the psychological literature (e.g. Davis \& Parasuraman 1982). Vigilance is not only a state of behavioural alertness to predators (e.g. Lima \& Dill 1990); rather, it is a general condition of enhanced ability to process information. Vigilance decrement therefore might cause either a gradual decrease in ability to notice an approaching camouflaged predator, a decline in capacity to detect cryptic prey, a reduced effectiveness in decision-making tasks, or more likely, some combination of all of the above.

For brevity, we distinguish here only between activity and rest, and assume that all activity is devoted to foraging and detecting predators. Regardless, our general arguments are relevant for other kinds of activity such as mate choice, nest building, or care of offspring.

(C) 1995 The A ssociation for the Study of A nimal Behaviour 1259 
Table I. Symbols used in the models

\begin{tabular}{|c|c|}
\hline Symbol & Description \\
\hline $\mathrm{t}$ & Time \\
\hline$v$ & Vigilance level \\
\hline $\bar{v}$ & Equilibrium vigilance level \\
\hline$\alpha$ & $\begin{array}{l}R \text { ate of vigilance decrement during foraging } \\
\text { activity }\end{array}$ \\
\hline$\beta$ & $\mathrm{R}$ ate of vigilance recovery during rest \\
\hline$\theta$ & $\begin{array}{l}\text { Proportion of time allotted to foraging } \\
\text { activity (foraging effort); } 0 \leq \theta \leq 1\end{array}$ \\
\hline$\theta_{0}^{*}$ & $\begin{array}{l}\text { The value of } \theta \text { that maximizes the rate of } \\
\text { food intake }\end{array}$ \\
\hline$\theta_{1}^{*}$ & $\begin{array}{l}\text { The value of } \theta \text { that maximizes expected } \\
\text { lifetime food intake }\end{array}$ \\
\hline$\theta_{\mathrm{m}}$ & A critical, break-even level of foraging effort \\
\hline$\lambda$ & A constant proportional to prey density \\
\hline $\bar{f}$ & Long-term average rate of food intake \\
\hline$\mu$ & Predation risk, per unit time, per predator \\
\hline $\bar{\mu}$ & Equilibrium predation risk \\
\hline$\mu_{\mathrm{r}}$ & M ortality risk, per unit time, while resting \\
\hline$\rho$ & Density of predators \\
\hline M & Overall mortality risk \\
\hline c & M etabolic cost, per unit time \\
\hline $\mathrm{E}$ & $\mathrm{N}$ et rate of energy gain \\
\hline $\mathrm{F}$ & Total expected lifetime energy gain \\
\hline$\tau$ & $\begin{array}{l}\text { Time delay between cessation of foraging } \\
\text { and onset of recovery of vigilance while } \\
\text { resting }\end{array}$ \\
\hline$v_{1}, v_{0}$ & $\begin{array}{l}\text { Vigilance levels at the beginning and end of } \\
\text { one foraging bout, respectively }\end{array}$ \\
\hline$t_{f}$ & Duration of one foraging bout \\
\hline$t_{f}^{*}$ & Optimal duration of a foraging bout \\
\hline$t_{r}$ & Duration of one resting period \\
\hline
\end{tabular}

\section{The Basic M odel}

First we model the dynamics of the forager's vigilance level $v=v(t)$ while foraging and resting alternately. For simplicity, we assume constant proportional rates of decline and recovery of $v$ as follows

$$
\frac{d v}{d t}= \begin{cases}-\alpha v & \text { while foraging } \\ \beta(1-v) & \text { while resting }\end{cases}
$$

where $\alpha, \beta$ are positive constants (T able I). H ere $\alpha$ is the rate of vigilance decrement, which is positively correlated with task difficulty (Parasuraman 1979; N uechterlein et al. 1983; Parasuraman \& Mouloua 1987). For example, attempting to detect targets that are more cryptic, or trying to attend to an increasing amount of sensory information simultaneously might both result in a higher rate of vigilance decrement. Similarly, $\beta$, the rate of recovery of vigilance, is determined by the quality of rest and sleep. F or example, rest periods with frequent interruptions for scanning the environment for predators (Lendrem 1983, 1984) might result in a lower rate of vigilance recovery (Horne 1988). N ote that equation (1) implies that the asymptotic level of vigilance while resting is $v=1$; this value merely scales the variable $v$.

Suppose that the forager allocates a portion $\theta$ of its time to foraging activity, and $(1-\theta)$ to resting. We refer to $\theta$ as foraging 'effort'. F irst, we suppose that each foraging or resting episode is of relatively short duration. This assumption, which we relax later, allows us to obtain a biologically relevant and simple analytic solution. The dynamics of the vigilance level is then given by

$$
\frac{d v}{d t}=-\theta \alpha v+(1-\theta) \beta(1-v)
$$

The equilibrium level of vigilance $v=\bar{v}(\theta)$ is calculated by setting $d v / d t=0$

$$
\bar{v}(\theta)=\frac{(1-\theta) \beta}{\theta \alpha+(1-\theta) \beta}
$$

A s anticipated, the equilibrium vigilance level $\bar{v}(\theta)$ is a decreasing function of foraging effort $\theta$, with $\bar{v}(0)=1$ and $\bar{v}(1)=0$. The equilibrium level of vigilance $\bar{v}(\theta)$ also depends on the ratio $\alpha / \beta$. F or a given level of foraging effort $\theta, \bar{v}(\theta)$ is lower for larger values of $\alpha / \beta$. Graphically, $\bar{v}(\theta)$ has a concave shape for values of $\alpha / \beta$ larger than 1 , that is, where the rate of vigilance decrement $(\alpha)$ is larger than the rate of recovery during rest $(\beta)$. For values of $\alpha / \beta$ lower than $1, \bar{v}(\theta)$ is convex (Fig. 1a).

\section{Predator-free F oraging}

We assume that the average rate of food intake while foraging is proportional to the current vigilance level

$$
\text { A verage rate of food intake }=\lambda v
$$

where $\lambda$ is a positive constant proportional to the density of prey. If the forager allocates proportion $\theta$ of its time to foraging, then the long-term average rate of food intake is

$$
\bar{f}(\theta)=\lambda \theta \bar{v}(\theta)
$$



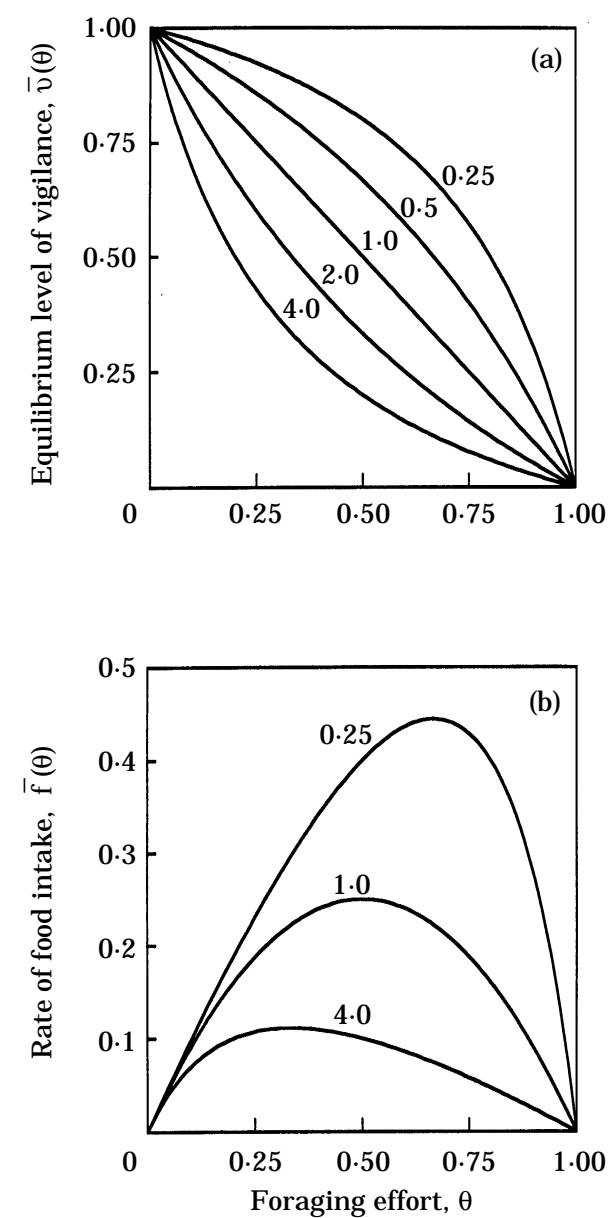

Figure 1. (a) Equilibrium level of vigilance $\bar{v}(\theta)$, as a function of the proportion of time allocated to foraging activity, $\theta$. Values of the ratio $\alpha / \beta$ of the rates of decline and recovery of vigilance during foraging and resting, respectively, are: $0.25,0.5,1 \cdot 0,2 \cdot 0,4 \cdot 0$. (b) Long-term average rate of foraging success, $\bar{f}(\theta)$. Values of $\alpha / \beta$ are: $0.25,1 \cdot 0,4 \cdot 0$

Increasing foraging effort $(\theta)$ has two effects. First, it increases the forager's rate of encountering prey, $\lambda \theta$. Second, it decreases the equilibrium level of vigilance, $\bar{v}(\theta)$. The combined outcome is an initial increase in rate of food intake, $\bar{f}(\theta)$, followed by a decline. Both the rates and magnitudes of increase and decrease are functions of the ratio $\alpha / \beta$ (Fig. 1b).

If we first neglect other aspects of fitness (such as predation risk and metabolic cost), it is reasonable to suppose that the optimal allocation of foraging effort, $\theta_{0}^{*}$, is the value of $\theta$ that maximizes the average rate of food intake $\bar{f}(\theta)$. F rom equations (3) and (5) it follows that $\theta_{0}^{*}$ maximizes

$$
\theta \bar{v}(\theta)=\frac{\theta(1-\theta) \beta}{\theta \alpha+(1-\theta) \beta}=\frac{1}{(\alpha / \beta) /(1-\theta)+1 / \theta}
$$

$M$ aximizing this expression is equivalent to minimizing its reciprocal, and a simple application of calculus then yields

$$
\theta_{0}^{*}=\frac{1}{1+\sqrt{\alpha / \beta}}
$$

U nder these assumptions, our model thus predicts that the optimal allocation of time between foraging activity and resting depends only on the ratio $\alpha / \beta$ of the rates of decline and recovery of vigilance $v$ while foraging or resting, respectively. F or example, if vigilance decrement is rapid relative to recovery $(\alpha \gg \beta)$ then the optimal strategy allocates a small fraction of the forager's time to foraging, and vice versa (Fig. 1b). The maximum long-term average rate of food intake is

$$
\bar{f}\left(\theta_{0}^{*}\right)=\frac{\lambda}{(1+\sqrt{\alpha / \beta})^{2}}
$$

A s expected, the average rate of food intake is a decreasing function of the ratio of the rates of vigilance decline and recovery $(\alpha / \beta)$, and an increasing function of prey density $(\lambda)$.

\section{E ffects of Predation R isk and M etabolic C ost}

We now extend the above model to allow for (1) risk of predation and (2) metabolic cost. A forager's vulnerability to predators may depend on its vigilance level. Let $\mu(v)$ denote predation risk (per unit time) from a single predator, while foraging, as a function of current vigilance level, v. We assume that an increased level of vigilance reduces predation risk, i.e. $d \mu / d v<0$. If $\rho$ denotes the abundance of predators, then overall predation risk equals $\rho \mu(v)$.

A ny given level of foraging effort $\theta$ implies an equilibrium level of vigilance, $\bar{v}(\theta)$, see equation (3), and a corresponding equilibrium predation risk $\bar{\mu}(\theta)$ while foraging

$$
\bar{\mu}(\theta)=\mu(\bar{v}(\theta))
$$

We have

$$
\frac{\mathrm{d} \bar{\mu}(\theta)}{\mathrm{d} \theta}>0
$$




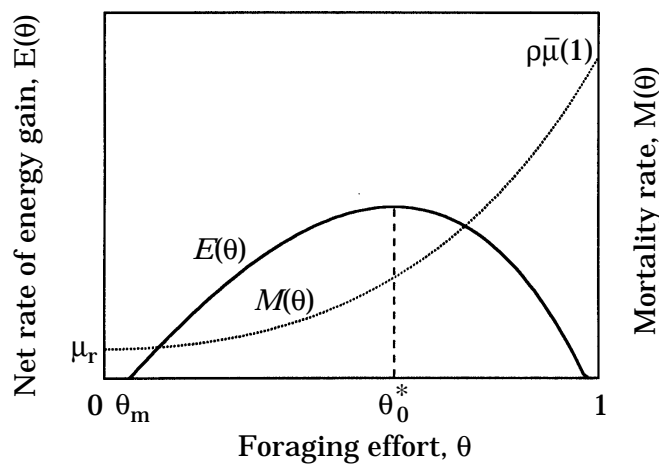

Figure 2. Net rate of energy gain $E(\theta)$, and overall mortality rate due to predation $M(\theta)$, as functions of foraging effort $\theta$. N et rate of energy gain equals 0 at $\theta_{m}$, where energy gain just balances metabolic costs. The maximum value of $E(\theta)$ is at $\theta_{0}^{*}$, which is the optimal foraging effort if predation risk is ignored. M ortality rate is at its minimum for $\theta=0$; this is the resting mortality rate $\mu_{r}$. From this point, mortality rate increases to its maximum at $\theta=1$. The overall optimal foraging effort is determined by equation (12) and depicted in Fig. 3.

Therefore, increases in foraging effort reduce the level of vigilance, and this results in increased predation risk (Fig. 2). Note again that we use 'vigilance' here in its broader psychological meaning, and that our argument is different from that of typical discussions of behavioural vigilance towards predators (reviewed in Lima \& Dill 1990). $\mathrm{Here}$, what we term 'foraging effort' already includes both feeding and scanning the environment for predators. This activity causes vigilance decrement and consequently, a reduced capacity to locate both food items and predators.

If $\mu_{r}$ denotes mortality risk (per unit time) while resting, the overall mortality risk associated with foraging effort $\theta$ is given by

$$
M(\theta)=\rho \theta \bar{\mu}(\theta)+(1-\theta) \mu_{\mathrm{r}}
$$

$\mathrm{N}$ ext, let $\mathrm{c}$ denote metabolic cost, per unit time. F or simplicity we suppose that metabolic cost is the same during foraging and resting activities. Thus the equilibrium average net rate of energy gain (assuming both $\bar{f}(\theta)$ and $c$ to be specified in terms of energy rates) becomes (see equation 5 )

$$
E(\theta)=\lambda \theta \bar{v}(\theta)-c
$$

The functions $E(\theta)$ and $M(\theta)$ are graphed, for typical parameter values, in $\mathrm{Fig}$. 2. $\mathrm{N}$ ote that $\mathrm{E}(\theta)$ reaches a maximum value at $\theta=\theta_{0}^{*}$ given by equation (6); $\theta_{0}^{*}$ is the optimal foraging effort if predation risk is ignored, and it depends only on the ratio $\alpha / \beta$. A Iso $E(\theta)<0$ for $\theta<\theta_{m}$ : unless foraging intensity is at least as large as this critical level $\theta_{\mathrm{m}}$ the forager cannot maintain a nonnegative energy balance. Given $\alpha / \beta$, the value of $\theta_{\mathrm{m}}$ depends only on the ratio $\lambda / c$; larger metabolic cost (relative to food abundance) increases the critical level of foraging effort, $\theta_{m}$.

In Fig. 2 the overall mortality risk $M(\theta)$ is shown as an increasing function of foraging effort $\theta$. This will be the case provided that resting mortality $\mu_{r}$ is less than the maximum predation risk $\rho \bar{\mu}(1)$, which is probably true in most circumstances. A ssuming that there are no other sources of mortality, total expected lifetime energy gain is then given by

$$
F(\theta)=\int_{0}^{\infty} e^{-M(\theta) t} E(\theta) d t=\frac{E(\theta)}{M(\theta)}
$$

To the extent that net energy gain transforms into lifetime reproductive success, $F(\theta)$ is a reasonable lifetime fitness currency. Thus the optimal level of foraging effort $\theta_{1}^{*}$ maximizes the ratio $E(\theta) / M(\theta)$, of net energy gain over mortality risk. A similar criterion, stated in terms of minimizing the ratio of mortality risk to growth rate, was used by Werner \& G illiam (1984) in studying ontogenetic habitat shifts (see also Clark 1994).

By calculus, the value $\theta=\theta_{1}^{*}$ that maximizes $F(\theta)=E(\theta) / M(\theta)$ satisfies $F^{\prime}(\theta)=0$, that is

$$
\frac{d}{d \theta}\left(\frac{E}{M}\right)=\frac{1}{M^{2}}\left(M \frac{d E}{d \theta}-E \frac{d M}{d \theta}\right)=0
$$

which can be rewritten as

$$
\frac{1}{\mathrm{E}} \frac{\mathrm{dE}}{\mathrm{d} \theta}=\frac{1}{\mathrm{M}} \frac{\mathrm{dM}}{\mathrm{d} \theta}
$$

The expression $(1 / E)(d E / d \theta)$ represents the proportional increase in energy gained (dE/E), resulting from a unit increase in foraging effort $(\mathrm{d} \theta=1$ unit). Thus, equation (12) asserts that the optimal trade-off between increased energy gain and increased mortality risk is achieved when the proportional increase in energy gain is just balanced by the proportional increased risk. The ratios $E^{\prime} / E$ and $M M^{\prime} / M$ (where $E^{\prime}$ is equal to $d E / d \theta$, etc.) are graphed in Fig. 3; the optimal foraging effort $\theta_{1}^{*}$ is determined by the point of intersection 


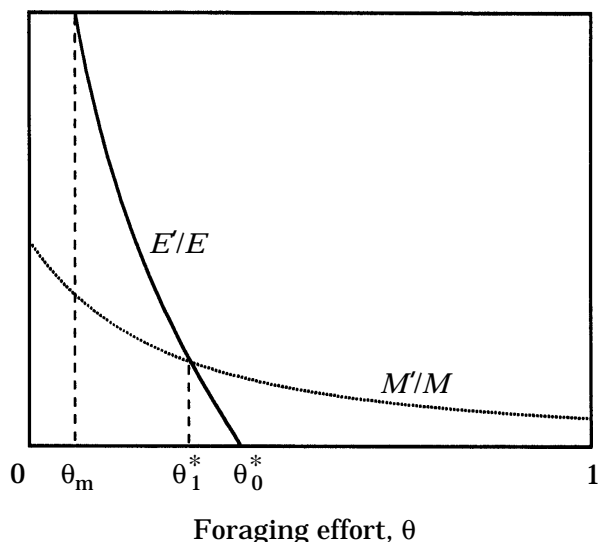

Figure 3. The ratios $E^{\prime}(\theta) / E(\theta)$ and $M^{\prime}(\theta) / M(\theta)$, as functions of foraging effort $\theta$. The optimal foraging effort $\theta_{1}^{*}$ is determined by the point of intersection of the two graphs. (See equation 12 and text for details and sensitivity analysis.)

of these two curves (equation 12). This optimal effort level is greater than $\theta_{m}$, the critical level of effort to maintain energy balance, and less than $\theta_{0}^{*}$, the level that maximizes net rate of energy gain, ignoring predation risk.

We can use equation (12) to study the sensitivity of optimal foraging effort $\theta_{1}^{*}$ (and hence also the sensitivity of the optimal level of vigilance $\bar{v}\left(\theta_{1}^{*}\right)$ ) to the following six model parameters: vigilance dynamics, $\alpha$ and $\beta$; prey abundance, $\lambda$; metabolic cost, c; predator abundance, $\rho$; resting mortality rate, $\mu_{r}$. It follows immediately from equations (9-11), however, that $F(\theta)$ does not depend on these six parameters separately, but only on the three ratios: (1) of the rates of decline and recovery of vigilance, $\alpha / \beta ;(2)$ of metabolic cost to prey abundance, $c / \lambda$; (3) of predator abundance to resting mortality risk, $\rho / \mu_{r}$. (In fact $F(\theta)$ also depends on the form of the predation-risk function $\mu(v)$, but we will not attempt to analyse this dependence here.)

F irst, on intuitive grounds it would seem that the optimal level of effort $\theta_{1}^{*}$ should decrease if the ratio $\alpha / \beta$ of vigilance decrement to vigilance recovery rates increases. While this is correct for $\theta_{0}^{*}$ in the simpler model (see Figs $1 \mathrm{~b}, 2$, and equation 6), the dependence of $F(\theta)$ on this ratio is more complex. In the special case that $\mu_{r}=0$ and $\bar{\mu}(\theta)$ is proportional to $\bar{v}(\theta)$ it is easy to see that $\theta_{1}^{*}$ does decrease as $\alpha / \beta$ increases, but the general case is unclear. Second, if the ratio $c / \lambda$ is increased, the vertical asymptote of $E^{\prime} / E$ at $\theta=\theta_{\mathrm{m}}$ moves to the right, while $\theta_{0}^{*}$ remains fixed. The entire $E^{\prime} / E$ curve moves to the right, causing the intersection $\theta_{1}^{*}$ also to move right, regardless of the position of the $M^{\prime} / M$ curve. The conclusion is intuitively reasonable: an increase in metabolic cost relative to prey density causes the forager to forage more intensively. (If $\mathrm{c} / \lambda$ is sufficiently large, however, we have $E(\theta)<0$ for all $\theta$; in this case the forager is unable to maintain positive energy balance. We do not treat this possibility here.) Finally, if the ratio $\rho / \mu_{r}$ is sufficiently high then $M{ }^{\prime} / M$ increases (for all $\theta$ ) as this ratio is increased, causing $\theta_{1}^{*}$ to decline (Fig. 3). A gain this is intuitive: under high predation risk the forager should reduce foraging effort and accept lower rates of energy gain.

\section{The 0 ptimal L ength of a F oraging B out}

Throughout the above discussion we tacitly assumed that the forager would rapidly switch from foraging to resting, thereby maintaining an approximately constant level of vigilance $\bar{v}(\theta)$. $U$ nless there is some cost associated with switching between the two modes of behaviour, it would in fact be optimal to maintain a constant level of vigilance by rapid switching. In practice, however, switching costs seem inevitable. For example, in order to reduce predation risks while resting, the forager may need to leave the foraging arena, which may delay the recovery of vigilance. U pon resuming foraging activity, the forager's rate of success may be low until it locates a prey-rich patch or re-learns how to detect cryptic prey (Dukas \& Clark, in press).

To allow for such costs of switching within our simple analytical model, we now suppose that each switch from foraging to resting involves a fixed time delay $\tau>0$ (Fig. 4). Vigilance remains constant during this delay, but subsequently begins to rise again. (The model could readily be modified to allow for an additional delay between resting and foraging, or for other types of switching cost, but the qualitative predictions would not change.) The optimal strategy of foraging and resting now involves two basic decision variables: $v_{1}$, the maximum level of vigilance attained at the start of the foraging bout, and $v_{0}$, the minimum vigilance level at the end of the foraging bout. While foraging, the vigilance level $v(t)$ decreases 


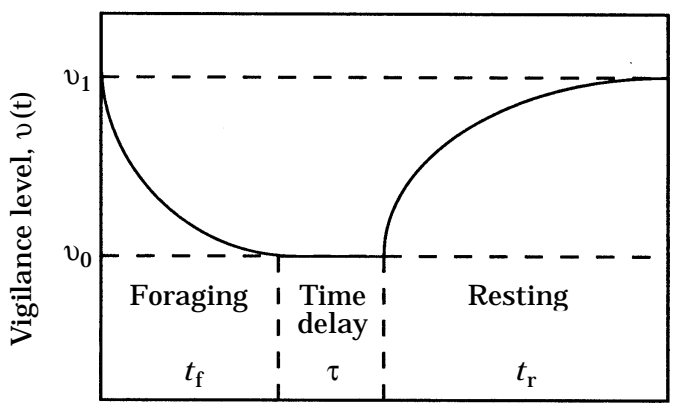

Time, $\mathrm{t}$

Figure 4. A graphic representation of parameters in the model predicting optimal length of a foraging bout, $t_{f}$. The length of a foraging bout is determined by maximizing equation (16) with respect to $v_{1}$, the maximum level of vigilance at the beginning of a foraging bout, and $v_{0}$, the level of vigilance at the end of the bout.

exponentially, as in equation (1): $v(t)=v_{1} e^{-\alpha t}$. Total food intake per foraging bout, of duration $t_{f}$, therefore equals

$$
F=\lambda \int_{0}^{t_{f}} v(t) d t=\frac{\lambda}{\alpha}\left(v_{1}-v_{0}\right)
$$

where $v_{0}=v_{1} \mathrm{e}^{-\alpha t_{f}}$ denotes the level of vigilance at the end of the bout. Thus

$$
t_{f}=\frac{1}{\alpha} \log \frac{v_{1}}{v_{0}}
$$

Similarly, the resting time $t_{r}$ required for vigilance to return from $v_{0}$ to the initial level $v_{1}$ is

$$
t_{r}=\frac{1}{\beta} \log \frac{1-v_{0}}{1-v_{1}}
$$

For simplicity, let us again ignore predation risk and metabolic cost. Then the long-term average rate of food intake, for a sequence of alternating bouts of foraging and resting, is given by

$$
\bar{F}\left(v_{0}, v_{1}\right)=\frac{\lambda}{\alpha} \frac{\left(v_{1}-v_{0}\right)}{t_{f}+t_{r}+\tau}
$$

where $\tau$ denotes the assumed delay between foraging and resting (Fig. 4). The optimal length of foraging bouts, $t_{f}^{*}$, is determined by maximizing this expression with respect to the two variables $v_{0}, v_{1}$. Figure 5 shows how the length of a foraging bout, $t_{f}^{*}$ depends on the parameter $\alpha$ (rate of vigilance decrement), and on the time delay $\tau$. As one would expect, rapid reduction of vigilance during foraging (high $\alpha$ ) implies that foraging

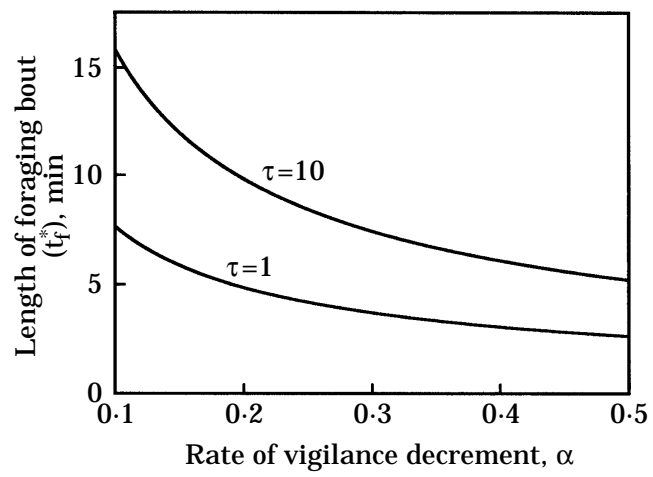

Figure 5. Optimal length of a foraging bout $\left(\mathrm{t}_{\mathrm{f}}^{*}\right)$ as a function of the rate of vigilance decrement $(\alpha)$, for two values of the cost of switching between foraging and resting $(\tau)$. H ere $\beta=0.012$ per min.

bouts should be short. However, if the cost of switching between foraging and resting is high (i.e. large $\tau$ ), then longer foraging bouts become optimal. On the other hand, if $\tau$ is negligibly small, the optimal length of a foraging bout, $t_{f}^{*}$, approaches zero, as we tacitly assumed in our first model.

\section{DISC USSIO N}

Essentially all animals show some temporal alternation between various kinds of activity, rest and sleep (e.g. Verbeek 1964; Herbers 1981; Horne 1988; A mlaner \& Ball 1989; $H$ artse 1989). These temporal patterns of activity have been proposed to be determined by factors such as forage availability, digestive capacity, predation risk and energy conservation (Wolf \& H ainsworth 1977; D aan 1981; D iamond et al. 1986; Elgar et al. 1988; Horne 1988; Lima \& D ill 1990). We suggest that vigilance decrement is an additional element with potentially strong effects on temporal patterns of behaviour.

Although a ubiquitous phenomenon, vigilance decrement is still not well understood at the neurobiological level. However, it has been associated with mechanisms such as habituation and neural inhibition (reviewed in $\mathrm{M}$ ackworth 1968, 1969). R egardless of the exact proximate mechanisms, it is widely agreed that vigilance decrement during continuous activity, and vigilance recovery during rest and sleep, do occur (Dimond \& Lazarus 1974; Jerison 1977; Davis \& 
Parasuraman 1982; Horne 1988; Steriade et al. 1993). F urthermore, probably everybody is familiar with the feeling of getting 'tired' or 'sleepy' while conducting some difficult activity, and then becoming 'refreshed' after rest or sleep. These are in fact, a lay person's depictions of the terms vigilance decrement and recovery, that we use here. The remarkable example of dolphins that sleep with one brain hemisphere at a time provides strong support for the notion of vigilance decrement and recovery. Dolphins sleep in the water but must continue to swim to the surface in order to breathe. Thus half-brain sleep seems to be an adaptation for balancing the simultaneous demands of vigilance recovery and continuous breathing (M ukhametov 1984; H orne 1988).

It is also commonly agreed that the rate of vigilance decrement is positively correlated with task difficulty (e.g. N uechterlein et al. 1983). We can distinguish between two related types of task difficulty. The first type can be defined based solely on the quality of external stimuli; for instance, making targets more similar to their surrounding background increases the difficulty of a detection task. The definition of the other type involves internal processing of information; for example, tasks that require subjects to process more information simultaneously are more difficult (Parasuraman 1979; Fisk \& Schneider 1981; Parasuraman \& M ouloua 1987; Dukas \& Ellner 1993). Similarly, attempting either to solve less familar problems, or to learn less familiar tasks is more difficult. $\mathrm{N}$ ote that although the above distinction is useful for practical purposes, it is likely that both types of tasks pertain to a similar process of elevated neural activity accompanied with fatigue.

\section{Predictions and Experimental D ata}

Our models predict that a larger value of the ratio $\alpha / \beta$ of vigilance decrement and recovery should decrease the proportion of time allocated to activity, and increase rest time (F igs $1 b, 2,3$ ). That is, for a given value of vigilance recovery rate $(\beta)$, the proportion of time devoted for rest should be an increasing function of the rate of vigilance decrement $(\alpha)$. As noted above, the rate of vigilance decrement is larger for more difficult tasks. Hence, our models predict increased rest time after more challenging activities such as learning new tasks or foraging for prey that is more difficult to detect or capture. This prediction is supported by some evidence from both human and non-human studies.

A s an example from human studies, $\mathrm{H}$ orne $\&$ $M$ inard (1985) provided subjects with extra stimulation on 1 out of 5 consecutive experimental days. The extra stimulation comprised a set of tours to unfamiliar localities, and activities such as visits to a zoo, an amusement park and a movie theatre. During the following night, subjects showed a significant increase in short-wave sleep compared with the other 4 nights. Other similar experimental results are discussed in Horne (1988).

We know of two relevant experiments using non-human subjects. First, Shaffery et al. (1985) provided experimental pairs of herring gull, $L$ arus argentatus, with a daily ration of $500 \mathrm{~g}$ of meat, and allowed control pairs to continue foraging naturally. F oraging is the most demanding activity conducted by the gulls and is much more difficult than staying on the territory. Shaffery et al. (1985) found that gulls that foraged naturally spent twice as much time in front-head sleep as the experimentally fed gulls. The experimental gulls, which were freed of the need for lengthy foraging activity, spent longer time on their breeding territories, engaged in the easy task of remaining alert for intruders. Second, Tobler (1983) forced cockroaches, L eucophea maderae, to be active during the last $3 \mathrm{~h}$ of the light period. She found that these experimental subjects increased resting time during the subsequent first hours of the dark period compared with control subjects.

In the above report of experimental results, we mentioned besides 'rest' also 'short-wave' and 'front-head' sleep. U nfortunately, we cannot yet interpret the exact relation between these and other kinds of rest and sleep. Neither do we know their specific effect on vigilance recovery. This reflects the current lack of sufficient understanding of rest and sleep mechanisms (H orne 1988; Amlaner \& Ball 1989; Hartse 1989; Steriade et al. 1993). N evertheless, the fact that all of the above studies have found a positive correlation between increased task difficulty and some kind of rest is in agreement with our predictions. In any event, although such experimental evidence is intriguing, it clearly cannot qualify as a test of our models. Thus it must be augmented by well-controlled studies that manipulate task difficulty and assess its effect on rest-activity schedule (see below). 
Despite the lack of knowledge about rest mechanisms, we can suggest one ecologically important association between quality of rest and vigilance recovery. Sleep in many birds involves a period of eye closure interrupted by 'peeks' of eye opening. Peeking allows a bird to scan the sleeping site for predators. Peeking rates and durations were monitored by Lendrem $(1983,1984)$ in two related studies involving field observations of mallards, Anas platyrhynchos, and experimental manipulations of Barbary doves, Streptopelia risoria. Lendrem found that several factors associated with elevated predation risk caused an increase in peeking rates. For instance, decreasing doves' group sizes from six to one caused a two-fold increase in individual peeking. While the advantage of peeking is obvious, the fact that it is always kept to some optimal minimum implies that it is costly. This cost of peeking is most likely a reduction in rate of vigilance recuperation.

\section{W ays of E valuating the M odels}

Although we centre here on effects of vigilance decrement on temporal patterns of behaviour, it is obvious that other factors such as food availability and digestive constraints may not be less important. It is therefore likely that a combination of, and interactions between several factors determine such temporal patterns. While this may somehow confine evaluating the relative importance of vigilance decrement for animals in the field, the principal assumptions and predictions of our models can be tested under controlled laboratory conditions.

First, our main assumption is that conducting a difficult and continuous activity results in vigilance decrement, and that the rate of decline is larger for more difficult tasks (Fig. 1a). This assumption has not been critically tested for non-human species. To test it, some excellent experimental paradigms with humans (e.g. N uechterlein et al. 1983; Parasuraman \& M ouloua 1987) can readily be modified for testing subjects such as pigeons or jays (e.g. Pietrewicz \& K amil 1981; Blough 1991). F or example, subjects should first learn to detect targets of three types differing in conspicuousness during several preliminary sessions. The difficulty of each of the three detection tasks would be determined in these preliminary sessions. The experimenter could evaluate difficulty by measuring the degree of similarity between each target type and its background. D ifficulty as perceived by subjects could be determined a priori by monitoring detection rates for each target at the start of a trial. N ext, the subjects would be required to detect items of one type in an experimental session followed by a rest period. In later sessions, the same subjects would be tested for detection of each of the other two target types. The order of sessions of 'easy', 'medium' and 'difficult' to detect target types should be assigned randomly to subjects. We predict no decline in target detection rate during a session for the easy task, little decline for the medium task, and a large decline for the difficult task ( $\mathrm{N}$ uechterlein et al. 1983).

Second, our basic (predation-free) model pre dicts that increased task difficulty would result in lower proportion of activity and a corresponding longer resting period. There are two ways to test this prediction. First, one can examine whether increased task difficulty is associated with a longer resting period. Such a study may be similar in general design to the field experiment of Shaffery et al. (1985) described above. Second, one may test whether increased task difficulty results in a shorter activity period. To examine this prediction with foragers, one must dissociate 'feeding' activity from aspects of food digestion and satiation. This can be done either by studying subjects that hoard food (D aan 1981; L ucas et al. 1993) or by using non-food reward. F or example, an artificial sweetener may be used in rat experiments (e.g. Capaldi 1991). Using a similar design, one can also test our prediction that the length of each foraging bout should be shorter for more difficult tasks (Fig. 5).

To conclude, we suggest that vigilance decre ment is a dominant factor determining temporal patterns of animal behaviour. While some of our models' predictions agree with previous experimental data, a more critical assessment of our assumptions and predictions can readily be made using the methods detailed above.

\section{A C KN O W LED G M ENTS}

We thank L. G ass, D. G rünbaum, N . W aser, M . West, D. Wilkie, R. Y denberg and two anonymous referees for helpful comments on the manuscript, and the B.C. mountains for helping us recuperate our vigilance. Our research is supported by N SER C (C anada: grant no. 83990). 


\section{REFERENCES}

A mlaner, C. J., J r \& Ball, N. J. 1989. A vian sleep. In: Principles and Practice of Sleep $M$ edicine ( $E d$. by M. H. K ryger, T. R oth \& W . C. D ement), pp. 50-63. Philadelphia: Saunders.

A ston-J ones, G., F oote, S. L. \& Bloom, F. E. 1984. A natomy and physiology of locus coeruleus neurons: functional implications. In: N orepinephrine (Ed. by M. G. Ziegler \& C. R. Lake), pp. 92-116. Baltimore: Williams \& Wilkins.

Blough, P. H. 1991. Selective attention and search images in pigeons. J. exp. Psychol. A nim. Behav. Proc., 17, 292-298.

Capaldi, E. D. 1991. H unger and the learning of flavor preferences. In: The $\mathrm{H}$ edonic of Taste $(\mathrm{Ed}$. by R. C. Bolles), pp. 127-142. Hillsdale, $\mathrm{N}$ ew J ersey: L awrence Erlbaum.

Clark, C. W. 1994. Antipredator behavior and the asset-protection principle. B ehav. E col., 5, 159-170.

$\mathrm{D}$ aan, S. 1981. A daptive daily strategies in behavior. In $\mathrm{H}$ andbook of Behavioral Neurobiology, $\mathrm{V}$ ol 4 (Ed. by J. A schoff), pp. 275-298. N ew Y ork: Plenum Press.

D avis, D. R . \& Parasuraman, R . 1982. The P sychology of $V$ igilance. $\mathrm{N}$ ew $\mathrm{Y}$ ork: A cademic Press.

D iamond, J. M ., K arasov, W. H., Phan, D .\& Carpenter, F. L. 1986. Digestive physiology is a determinant of foraging bout frequency in hummingbirds. Nature, L ond., 320, 62-63.

Dimond, S. \& Lazarus, J. 1974. The problem of vigilance in animal life. Brain Behav. Evol., 9, 60-79.

D ukas, R . \& Clark, C. W. In press. Searching for cryptic prey: a dynamic model. E cology.

Dukas, R. \& Ellner, S. 1993. Information processing and prey detection. E cology, 74, 1337-1346.

Elgar, M. A., Pagel, M. D. \& Harvey, P. H . 1988. Sleep in mammals. A nim. B ehav., 36, 1407-1419.

Fisk, A. D. \& Schneider, W. 1981. Control and automatic processing during tasks requiring sustained attention: a new approach to vigilance. $\mathrm{H}$ um. Factors, 23, 737-750.

F oote, S. L., A ston-J ones, G.\& Bloom, F. E. 1980. Impulse activity of locus coeruleus neurons in awake rats and monkeys is a function of sensory stimulation and arousal. P roc. natn. A cad. Sci. U.S.A., 77, 3033-3037.

$\mathrm{H}$ artse, K. M . 1989. Sleep in insects and nonmammalian vertebrates. In: P rinciples and P ractice of Sleep M edicine (E d. by M . H. K ryger, T. R oth \& W . C. D ement), pp. 64-73. Philadelphia: Saunders.

Herbers, J. M. 1981. Time resources and laziness in animals. O ecologia ( Berl.) , 49, 252-262.

Horne, J. 1988. Why We Sleep. Oxford: Oxford U niversity Press.

Horne, J. \& M inard, A. 1985. Sleep and sleepiness following a behaviourally 'active' day. Ergonomics, 28, 567-575.

Jerison, H. J. 1977. Vigilance: biology, psychology, theory and practice. In: V igilance: Theory, 0 perational Performance and Physiological Correlates ( $\mathrm{Ed}$. by R. R. M ackie), pp. 27-40. N ew Y ork: Plenum Press.

L endrem, D. W. 1983. Sleeping and vigilance in birds. I. Field observations of the mallard (A nas platyrhynchos). A nim. B ehav., 31, 532-538.
Lendrem, D. W. 1984. Sleeping and vigilance in birds. II. An experimental study of the Barbary doves (Streptopelia risoria). A nim. B ehav., 32, 243-248.

Lima, S. L. \& Dill, L. M. 1990. Behavioral decisions made under the risk of predation: a review and prospectus. Can. J . Z ool., 68, 619-640.

Lucas, J. R., Peterson, L. J . \& Boudinier, R . L. 1993. The effects of time constraints and changes in body mass and satiation on the simultaneous expression of caching and diet-choice decisions. A nim. B ehav., 45, 639-658.

Mackie, R. R. 1977. Vigilance: Theory, O perational Performance and P hysiological Correlates. $\mathrm{N}$ ew $\mathrm{Y}$ ork: Plenum Press.

M ackworth, J. F. 1968. Vigilance, arousal and habituation. P sychol. R ev., 75, 308-322.

M ackworth, J. F. 1969. Vigilance and $\mathrm{H}$ abituation a Neuropsychological Approach. Harmondsworth: Penguin.

M ackworth, N. H. 1948. The breakdown of vigilance during prolonged visual search. Q. J I exp. P sychol., 1 , 6-21.

M ukhametov, L. M. 1984. Sleep in marine mammals. In: Sleep M echanisms (Ed. by A. A. Borbely \& J. L. Valatx), pp. 227-238. M unich: Springer.

N uechterlein, K . H ., Parasuraman, R . \& J iang, Q. 1983. $V$ isual sustained attention: image degradation produces rapid sensitivity decrement over time. Science 220, 327-329.

Parasuraman, R. 1979. M emory load and event rate control sensitivity decrements in sustained attention. Science, 205, 924-927.

Parasuraman, R. 1984. Sustained attention in detection and discrimination. In: Varieties of A ttention ( $E d$. by R. Parasuraman \& D. R. D avis), pp. 243-271. N ew Y ork: A cademic Press.

Parasuraman, R. \& M ouloua, M. 1987. Interaction of signal discriminability and task type in vigilance decrement. Percept. P sychophysiol., 41, 17-22.

Pietrewicz, A. T. \& K amil, A. C. 1981. Search images and the detection of cryptic prey: an operant approach. In: Foraging Behavior (Ed. by A. C. K amil $\&$ T. D. Sargent), pp. 311-331. N ew Y ork: G arland.

Shaffery, J. P., Ball, N.J. \& A mlaner, C. J., J r. 1985. $M$ anipulating daytime sleep in herring gulls (L arus argentatus). A nim. B ehav., 33, 566-572.

Steriade, M., M cCormick, D. A.\& Sejnowski, T. J. 1993. Thalamocortical oscillations in the sleeping and aroused brain. Science, 262, 679-685.

Tobler, I. 1983. Effects of forced locomotion on the rest-activity cycle of the cockroach. B ehav. B rain Res., 8, 351-360.

Verbeek, N. A. M. 1964. A time and energy budget study of the Brewer's blackbird. Condor, 66, 70-74.

Warm, J. S. 1984. Sustained A ttention in $\mathrm{H}$ uman Performance. N ew Y ork: J ohn Wiley.

Werner, E. E. \& Gilliam, J. F. 1984. The ontogenetic niche and species interactions in size-structured populations. A. R ev. Ecol. Syst., 15, 393-425.

Wickens, C. D. 1984. Engineering Psychology and H uman Performance. Columbus, Ohio: Bell \& H owell.

Wolf, L. L. \& H ainsworth, F. R. 1977. Temporal patterning of feeding by hummingbirds. A nim. B ehav., 25, 976-989. 